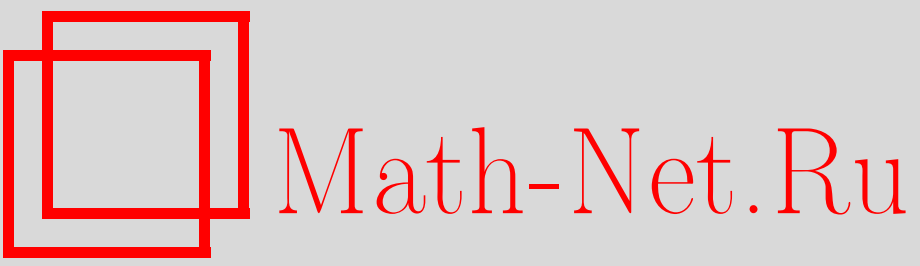

Д. В. Белова, Об одной смешанной задаче с инволюцией, Итоги науки и техн. Сер. Соврем. мат. и ее прил. Темат. обз., 2021, том 194, 46-54

DOI: https://doi.org/10.36535/0233-6723-2021-194-46-54

Использование Общероссийского математического портала Math-Net.Ru подразумевает, что вы прочитали и согласны с пользовательским соглашением

http://www.mathnet.ru/rus/agreement

Параметры загрузки:

IP: 54.162 .27 .143

26 апреля 2023 г., 11:32:26 


\title{
ОБ ОДНОЙ СМЕШАННОЙ ЗАДАЧЕ С ИНВОЛЮЦИЕЙ
}

\author{
(c) 2021 г. Д. В. БЕЛОВА
}

\begin{abstract}
АннотАция. В работе исследуется смешанная задача для уравнения с инволютивным отклонением в аргументе и с периодическими краевыми условиями. Методом Фурье получено классическое решение задачи при минимальных требованиях на начальные данные задачи. При этом использованы приемы по ускорению сходимости ряда, представляющего формальное решение.
\end{abstract}

Ключевые слова: функционально-дифференциальный оператор, инволюция, смешанная задача, метод Фурье.

\section{ON ONE MIXED PROBLEM WITH INVOLUTION}

\author{
(c) 2021 D. V. BELOVA
}

\begin{abstract}
In this paper, we examine a mixed problem for an equation with an involutive deviation in the argument and periodic boundary conditions. Using the Fourier method, we obtain a classical solution to the problem with minimal requirements for the initial data of the problem. Also, we used some methods of improving the convergence of the series representing a formal solution.
\end{abstract}

Keywords and phrases: functional differential operator, involution, mixed problem, Fourier method.

AMS Subject Classification: 35F16, 34K08

1. Введение. Рассматривается следующая смешанная задача с инволюцией и с периодическими краевыми условиями:

$$
\begin{gathered}
\frac{\partial u(x, t)}{\partial t}=\frac{\partial u(x, t)}{\partial x}+q_{1}(x) u(1-x, t)+q_{2}(x) u(x, t), \quad x \in[0 ; 1], \quad t \in(-\infty ;+\infty), \\
u(0, t)=u(1, t), \quad u(x, 0)=\varphi(x) .
\end{gathered}
$$

Предполагаем, что $q_{j}(x)$ и $\varphi(x)$ - комплекснозначные функции, $q_{j} \in C^{1}[0,1], j=1,2$, а на $\varphi(x)$ накладываем минимальные требования, следующие из постановки задачи: $\varphi \in C^{1}[0,1]$ и

$$
\varphi(0)=\varphi(1), \quad \varphi^{\prime}(0)-\varphi^{\prime}(1)+\varphi(0)\left[q_{1}(0)-q_{1}(1)+q_{2}(0)-q_{2}(1)\right]=0 .
$$

Уравнение (1) представляет собой простейшее уравнение в частных производных, содержащее инволюцию в аргументе $u(x, t)$, т.е. отображение $g(x)=1-x$ такое, что $g(g(x))=x$. Интерес к операторам с такой инволюцией вызван, в частности, работами А. П. Хромова (см. [14]) по исследованию интегральных операторов с инволюцией в верхнем пределе. Различные спектральные задачи для интегральных и функционально-дифференциальных операторов с инволюциями активно исследуются (см., например, $[1,8,10,12,15,16]$ и библиографию в них). Изучение смешанных задач для уравнения первого порядка с инволюцией проводится в $[2,4,6,7,9]$. Здесь будет использоваться техника работ $[4,6,6,7,9]$, основанная на применении метода Фурье и идей по ускорению сходимости ряда, представляющего формальное решение. В [7] исследовалась задача с периодическими краевыми условиями, но с инволюцией в производной (здесь иначе выбирается вспомогательная эталонная задача). Данная работа обобщает результаты работы [9]. 
Метод Фурье в задаче (1)-(2) приводит к спектральной задаче

$$
L y=\lambda y
$$

для оператора $L$ :

$$
(L y)(x)=y^{\prime}(x)+q_{1}(x) y(1-x)+q_{2}(x) y(x), \quad y(0)=y(1) .
$$

Наряду с $L$ будем также рассматривать оператор $L_{0}$, который получается из $L$ при $q_{1}(x)=0$ :

$$
\left(L_{0} y\right)(x)=y^{\prime}(x)+q_{2}(x) y(x), \quad y(0)=y(1) .
$$

Считаем, что область определения этих операторов $D_{L}\left(D_{L_{0}}\right)$ включает непрерывно-дифференцируемые функции, удовлетворяющие условиям $y(0)=y(1)$.

2. Эталонная задача. Задачу $(1)-(2)$ с $q_{1}(x) \equiv 0$ будем называть эталонной (в соответствии с [7]), ее решение будем обозначать $u_{0}(x, t)$. В этом случае уравнение $(1)$ является простейшим уравнением в частных производных первого порядка, и исследование задачи не представляет трудностей. Приведем здесь решение этой задачи методом Фурье, необходимое для исследования общего случая, показав возможность суммирования соответствующего формального ряда. Здесь достаточно предполагать, что $q_{2}(x) \in C[0,1]$. Условия (3) здесь заменяются на

$$
\varphi(0)=\varphi(1), \quad \varphi^{\prime}(0)-\varphi^{\prime}(1)+\varphi(0)\left[q_{2}(0)-q_{2}(1)\right]=0 .
$$

Решая соответствующую спектральную задачу $L_{0} y=\lambda y$, легко получаем следующее утверждение.

Лемма 1. Собственные значения $\lambda_{n}^{0}$ и собственные функции $y_{n}^{0}(x)$ оператора $L_{0}$ имеют вид

$$
\lambda_{n}^{0}=2 \pi n i+a, \quad y_{n}^{0}(x)=s(x) e^{2 \pi n i x}, \quad n \in \mathbb{Z},
$$

где

$$
a=\int_{0}^{1} q_{2}(t) d t, \quad s(x)=\exp \left(a x-\int_{0}^{x} q_{2}(t) d t\right) .
$$

Оператор $L_{0}$ не является самосопряженным. Собственному значению $\lambda_{n}^{0 *}=\bar{\lambda}_{n}^{0}$ сопряженного оператора

$$
\left(L_{0}^{*} z\right)(x)=-z^{\prime}(x)+\overline{q_{2}(x)} z(x), \quad z(0)=z(1),
$$

соответствует собственная функция $z_{n}(x)=e^{2 \pi n i x} s_{1}(x)$, где

$$
s_{1}(x)=\exp \left(-\bar{a} x+\int_{0}^{x} \overline{q_{2}(t)} d t\right) .
$$

Учитывая, что

$$
s_{1}(x)=\exp \left(\overline{-a x+\int_{0}^{x} q_{2}(t) d t}\right)=(\overline{s(x)})^{-1},
$$

получаем следующий результат.

Лемма 2. Собственные значения $\lambda_{n}^{0 *}$ и собственные функции $z_{n}^{0}(x)$ оператора $L_{0}^{*}$ имеют вид

$$
\lambda_{n}^{0^{*}}=-2 \pi n i+\bar{a}, \quad z_{n}^{0}(x)=(\overline{s(x)})^{-1} e^{2 \pi n i x}, \quad n \in \mathbb{Z},
$$

где а и $s(x)$ такие же, как в лемме 1.

Всюду далее через $(\cdot, \cdot)$ будем обозначать скалярное произведение в $L_{2}[0,1]$.

Лемма 3. Системь $\left\{y_{n}^{0}(x)\right\} u\left\{z_{n}^{0}(x)\right\}$ полны в $L_{2}[0,1]$, причем $\left(y_{n}^{0}, z_{n}^{0}\right)=1$. 
Доказательство. Пусть функция $f(x) \in L_{2}[0,1]$ ортогональна $\left\{y_{n}^{0}(x)\right\}$. Тогда

$$
\left(f, y_{n}^{0}\right)=\int_{0}^{1} f(x) \overline{s(x)} e^{-2 \pi n i x} d x=0
$$

откуда в силу полноты тригонометрической системы $\left\{e^{2 \pi n i x}\right\}$ получаем $f(x) \overline{s(x)} \equiv 0$ п. в. Отсюда следует: $f(x) \equiv 0$ п.в., что и доказывает полноту. Доказательство для $\left\{z_{n}^{0}(x)\right\}$ аналогично. Равенство $\left(y_{n}^{0}, z_{n}^{0}\right)=1$ очевидно.

Теорема 1. Если $q_{1}(x)=0, q_{2}(x) \in C[0,1], \varphi(x) \in C^{1}[0,1]$ и удовлетворяет условиям (5), то классическое решение задачи (1)-(2) существует и имеет вид

$$
u_{0}(x, t)=s(x) f_{0}(x+t) e^{a t},
$$

где $f_{0}(x) \in C^{1}(-\infty ;+\infty)$ - периодическая с периодом 1 функиия, причем $f_{0}(x)=\varphi(x) s^{-1}(x)$ при $x \in[0,1]$, а и $s(x)$ из леммы 1 .

Доказательство. Согласно методу Фурье решением задачи является формальный ряд

$$
u(x, t)=\sum_{n=-\infty}^{+\infty} c_{n} y_{n}^{0}(x) e^{\lambda_{n}^{0} t} .
$$

Подставляя в начальное условие $(2)$, с учетом леммы 3 находим $c_{n}=\left(\varphi, z_{n}^{0}\right)$. Таким образом, при $x \in[0,1]$ имеем

$$
u(x, 0)=s(x) \sum_{n=-\infty}^{+\infty}\left(\varphi, z_{n}^{0}\right) e^{2 \pi n i x} .
$$

Ряд в (7) определен при всех $x$. Обозначим его сумму через $f_{0}(x)$ :

$$
f_{0}(x)=\sum_{n=-\infty}^{+\infty}\left(\varphi, z_{n}^{0}\right) e^{2 \pi n i x}, \quad x \in(-\infty,+\infty) .
$$

Очевидно, что $f_{0}(x)$ - периодическая функция с периодом 1 . Так как

$$
\left(\varphi, z_{n}^{0}\right)=\left(\varphi,(\overline{s(x)})^{-1} e^{2 \pi n i x}\right)=\left(\varphi(x) s^{-1}(x), e^{2 \pi n i x}\right),
$$

и функция $\varphi(x) s^{-1}(x) \in D_{L_{0}}$ (из того, что $\left.s(0)=s(1)=1\right)$, то нетрудно показать, что этот ряд равномерно сходится при всех $x \in(-\infty,+\infty)$, а при $x \in[0,1]$ в силу полноты тригонометрической системы, его сумма равна $\varphi_{1}(x)=\varphi(x) / s(x)$. Таким образом, $f_{0}(x)=\varphi_{1}(x)$, при $x \in[0,1]$, а на остальной части всей числовой оси $f_{0}(x)$ определяется периодическим продолжением.

Исследуем гладкость $f_{0}(x)$. Из вышесказанного следует непрерывная дифференцируемость $f_{0}(x)$ всюду, кроме точек $x=k, k \in \mathbb{Z}$. Достаточно проверить, что

$$
f_{0}(0-0)=f_{0}(0+0), \quad f_{0}^{\prime}(0-0)=f_{0}^{\prime}(0+0) .
$$

Первое соотношение легко следует из периодичности $f_{0}(x)$, условий (5) и $s(0)=s(1)=1$. Далее, при $x \in[0,1]$ имеем

Поэтому

$$
f_{0}^{\prime}(x)=\varphi_{1}^{\prime}(x)=\frac{\varphi^{\prime}(x)-\varphi(x)\left(a-q_{2}(x)\right)}{s(x)} .
$$

$$
\begin{gathered}
f_{0}^{\prime}(0-0)=f_{0}^{\prime}(1-0)=\varphi_{1}^{\prime}(1)=\varphi^{\prime}(1)-\varphi(1)\left(a-q_{2}(1)\right), \\
f_{0}^{\prime}(0+0)=\varphi_{1}^{\prime}(0)=\varphi^{\prime}(0)-\varphi(0)\left(a-q_{2}(0)\right),
\end{gathered}
$$

и равенство $f_{0}^{\prime}(0-0)=f_{0}^{\prime}(0+0)$ следует из (5). Следовательно, $f_{0}(x) \in C^{1}(-\infty,+\infty)$.

Преобразуя формальный ряд, теперь его можно просуммировать:

$$
u(x, t)=\sum_{n=-\infty}^{+\infty}\left(\varphi_{1}, e^{2 \pi n i x}\right) s(x) e^{a t} e^{2 \pi n i(x+t)}=s(x) e^{a t} \sum_{n=-\infty}^{+\infty}\left(\varphi_{1}, e^{2 \pi n i x}\right) e^{2 \pi n i(x+t)},
$$


откуда получаем (6). Непосредственной проверкой можно убедиться, что (6) удовлетворяет уравнению (1) при $q_{1}(x) \equiv 0$ и условиям $(2)$.

3. Асимптотика собственных значений и собственных функций оператора $L$. Получим теперь асимптотические формулы для собственных значений и собственных функций оператора $L$. Так же как в [7] решение спектральной задачи (4) сводится к системе Дирака, а именно справедлива следующая лемма.

Лемма 4. Число $\lambda$ является собственным значением, а $y(x)$ собственной функиией краевой задачи (4) тогда и только тогда, когда $u(x)=\left(u_{1}(x), u_{2}(x)\right)^{T}=(y(x), y(1-x))^{T}($ здесъ $T-$ знак транспонирования) является ненулевым решением системы

$$
u^{\prime}(x)+Q(x) u(x)=\lambda D u(x)
$$

где

с краевыми условиями

$$
D=\left(\begin{array}{rr}
1 & 0 \\
0 & -1
\end{array}\right), \quad Q(x)=\left(\begin{array}{cc}
q_{2}(x) & q_{1}(x) \\
-q_{1}(1-x) & -q_{2}(1-x)
\end{array}\right)
$$

$$
u_{1}(0)=0, \quad u_{1}(1 / 2)=u_{2}(1 / 2)
$$

С помощью замены

$$
u_{1}(x)=h_{1}(x) z_{1}(x), \quad u_{2}(x)=h_{2}(x) z_{2}(x)
$$

где

$$
h_{1}(x)=\exp \left(-\int_{0}^{x} q_{2}(t) d t\right), \quad h_{2}(x)=\exp \left(-\int_{0}^{x} q_{2}(1-t) d t\right)
$$

система (8) сводится к системе Дирака

$$
z^{\prime}(x)+P(x) z(x)=\lambda D z(x)
$$

где

$$
P(x)=\left(\begin{array}{cc}
0 & p_{1}(x) \\
p_{2}(x) & 0
\end{array}\right), \quad D=\operatorname{diag}(1,-1), \quad p_{1}(x)=\frac{q_{1}(x) h_{2}(x)}{h_{1}(x)}, \quad p_{2}(x)=\frac{q_{1}(1-x) h_{1}(x)}{h_{2}(x)} .
$$

Уточненные асимптотические формулы для общего решения системы (11) в случае $p_{k} \in C^{1}[0,1]$ приведены, например в [7] (см. также [3]). Из [7, теорема 4] и (10) получаем следующее утверждение.

Лемма 5. Если $\operatorname{Re} \lambda \geqslant 0, q_{j}(x) \in C^{1}[0,1]$, то для общего решения уравнения (8) справедлива асимптотическая формула

$$
u(x, \lambda)=H(x) Z(x, \lambda) e^{\lambda D x} c
$$

где

$$
H(x)=\left(\begin{array}{cc}
h_{1}(x) & 0 \\
0 & h_{2}(x)
\end{array}\right), \quad Z(x, \lambda)=\left(z_{i j}(x, \lambda)\right)_{i, j=1,2},
$$

$c=\left(c_{1}, c_{2}\right)^{T}-$ произвольный вектор и

$$
\begin{aligned}
& z_{11}(x, \lambda)=1+\frac{1}{2 \lambda} \int_{0}^{x} p_{1}(t) p_{2}(t) d t+O\left(\frac{1}{\lambda^{2}}\right) \\
& z_{12}(x, \lambda)=\frac{1}{2 \lambda}\left(p_{2}(x)-p_{2}(1) e^{-2 \lambda(1-x)}+\int_{x}^{1} e^{2 \lambda(x-t)} p_{2}^{\prime}(t) d t\right)+O\left(\frac{1}{\lambda^{2}}\right),
\end{aligned}
$$




$$
\begin{aligned}
& z_{21}(x, \lambda)=-\frac{1}{2 \lambda}\left(p_{1}(x)-p_{1}(0) e^{-2 \lambda x}-\int_{0}^{x} e^{-2 \lambda(x-t)} p_{1}^{\prime}(t) d t\right)+O\left(\frac{1}{\lambda^{2}}\right) \\
& z_{22}(x, \lambda)=1-\frac{1}{2 \lambda} \int_{0}^{x} p_{1}(t) p_{2}(t) d t+O\left(\frac{1}{\lambda^{2}}\right) .
\end{aligned}
$$

Из леммы 5 и условий (9) получаем следующее уравнение для собственных значений:

$$
\left|\begin{array}{cc}
h_{1}(0) z_{11}(0)-h_{2}(0) z_{21}(0) & h_{1}(0) z_{12}(0)-h_{2}(0) z_{22}(0) \\
{\left[h_{1}\left(\frac{1}{2}\right) z_{11}\left(\frac{1}{2}\right)-h_{2}\left(\frac{1}{2}\right) z_{21}\left(\frac{1}{2}\right)\right] e^{\frac{\lambda}{2}}} & {\left[h_{1}\left(\frac{1}{2}\right) z_{12}\left(\frac{1}{2}\right)-h_{2}\left(\frac{1}{2}\right) z_{22}\left(\frac{1}{2}\right)\right] e^{-\frac{\lambda}{2}}}
\end{array}\right|=0
$$

(аргумент $\lambda$ у $z_{i j}$ для удобства опускаем). Используя «грубую» асимптотику для $z_{i j}$ из леммы 5 :

$$
z_{11}(x, \lambda)=1+O\left(\frac{1}{\lambda}\right), \quad z_{12}(x, \lambda)=O\left(\frac{1}{\lambda}\right), \quad z_{21}(x, \lambda)=O\left(\frac{1}{\lambda}\right), \quad z_{22}(x, \lambda)=1+O\left(\frac{1}{\lambda}\right),
$$

получим следующее утверждение.

Лемма 6. Для собственных значений и собственных функиий оператора $L$ имеют место следующие асимптотические формуль:

$$
\lambda_{n}=2 \pi n i+a+O\left(n^{-1}\right), \quad y_{n}(x)=s(x) e^{2 \pi n i x}+O\left(n^{-1}\right),
$$

где а и $s(x)$ из леммы $1, n= \pm n_{0}, \pm\left(n_{0}+1\right), \pm\left(n_{0}+2\right), \ldots, n_{0}$-некоторое достаточно большое число.

Следующая лемма аналогична [7, лемма 12].

Лемма 7. При $\lambda=\lambda_{n}$ для значений функций $z_{i j}(x, \lambda)$ из леммы 5 справедливы следующие асимптотические формуль:

$$
\begin{gathered}
z_{11}(0)=1+O\left(\frac{1}{n^{2}}\right), \quad z_{12}(0)=\frac{\alpha}{n}+\frac{\alpha_{n}}{n}+O\left(\frac{1}{n^{2}}\right), \\
z_{22}(0)=1+O\left(\frac{1}{n^{2}}\right), \quad z_{21}(0)=O\left(\frac{1}{n^{2}}\right), \\
z_{11}\left(\frac{1}{2}\right)=1+\frac{\alpha}{n}+O\left(\frac{1}{n^{2}}\right), \quad z_{12}\left(\frac{1}{2}\right)=\frac{\alpha}{n}+\frac{\alpha_{n}}{n}+O\left(\frac{1}{n^{2}}\right), \\
z_{22}\left(\frac{1}{2}\right)=1+\frac{\alpha}{n}+O\left(\frac{1}{n^{2}}\right), \quad z_{21}\left(\frac{1}{2}\right)=\frac{\alpha}{n}+\frac{\alpha_{n}}{n}+O\left(\frac{1}{n^{2}}\right)
\end{gathered}
$$

(аргумент $\lambda_{n}$ для удобства опускаем). Здесъ и всюду далее через а обозначаются различные константы, не зависящие от $n$ (из конечного набора констант), через $\alpha_{n}$-такие константы, что

$$
\sum_{-\infty}^{\infty}\left|\alpha_{n}\right|^{2}<\infty
$$

Используя теперь в (12) асимптотику из леммы 7, получаем следующую теорему.

Теорема 2. Для собственных значений и собственных функиий оператора L имеют место следующие уточненные асимптотические формуль:

где

$$
\begin{gathered}
\lambda_{n}=2 \pi n i+a+\frac{\alpha}{n}+\frac{\alpha_{n}}{n}+O\left(\frac{1}{n^{2}}\right), \\
y_{n}(x)=s(x) e^{2 \pi n i x}+\psi_{1 n}(x)+\psi_{2 n}(x)+O\left(\frac{1}{n^{2}}\right),
\end{gathered}
$$

$$
\psi_{1 n}(x)=\frac{1}{n}\left[b_{1}(x) e^{-\lambda_{n}^{0} x}+b_{2}(x) e^{\lambda_{n}^{0} x}+b_{3}(x) \alpha_{n} e^{-\lambda_{n}^{0} x}+b_{4}(x) \alpha_{n} e^{\lambda_{n}^{0} x}\right]
$$




$$
\psi_{2 n}(x)=\frac{1}{n}\left[b_{5}(x) \int_{0}^{x} e^{-\lambda_{n}^{0} t} q_{1}^{\prime}\left(\frac{x+t}{2}\right) d t+b_{6}(x) \int_{0}^{x} e^{\lambda_{n}^{0} t} q_{1}^{\prime}\left(\frac{x-t}{2}\right) d t\right],
$$

$b_{k}(x)$ - некоторые непрерывные функции.

Точный вид функций $b_{k}(x)$ не важен, достаточно знать, что $b_{k}(x) \in C[0,1]$.

Замечание. Аналогичную асимптотику получим для собственных значений $\lambda_{n}^{*}$ и собственных функций $z_{n}(x)$ сопряженного оператора (только главную часть асимптотик теперь будут давать $\lambda_{n}^{0 *}$ и $z_{n}^{0}(x)$ из леммы 2$)$.

4. Решение задачи (1)-(2). Согласно методу Фурье формальное решение задачи представим в виде (см. [13]):

$$
u(x, t)=-\frac{1}{2 \pi i}\left(\int_{\lambda \mid=r}+\sum_{|n| \geqslant n_{0} \gamma_{n}} \int_{\lambda}\right)\left(R_{\lambda} \varphi\right)(x) e^{\lambda t} d \lambda,
$$

где $R_{\lambda}=(L-\lambda E)^{-1}$ - резольвента оператора $L(E$ - единичный оператор, $\lambda$ - спектральный параметр), $\gamma_{n}=\{\lambda|| \lambda-2 \pi n i-a \mid=\delta\}, \delta>0$ и достаточно мало, чтобы собственные значения $\lambda_{n}$ попадали по одному внутрь $\gamma_{n}$, а $r>0$ фиксировано и таково, что все собственные значения, для которых $|n| \leqslant n_{0}$, попадают в контур $|\lambda|=r$. При этом

$$
-\frac{1}{2 \pi i} \int_{\gamma_{n}}\left(R_{\lambda} \varphi\right)(x) e^{\lambda t} d \lambda=\frac{\left(\varphi, z_{n}\right)}{\left(y_{n}, z_{n}\right)} y_{n}(x) e^{\lambda_{n} t} .
$$

По теореме 2 и замечанию к ней коэффициенты $c_{n}=\left(\varphi, z_{n}\right) /\left(y_{n}, z_{n}\right)=\alpha_{n} / n$, откуда в силу ограниченности $y_{n}(x)$ и $e^{\lambda_{n} t}$ следует равномерная сходимость ряда. Но эта оценка недостаточна для того, чтобы ряд можно было почленно дифференцировать. Следуя схеме работ [6,9], классическое решение может быть получено, но при дополнительных ограничениях на значения $q_{1}(x)$ в точках 0 и 1 (а именно, $\left.q_{1}(0)=q_{1}(1)\right)$. Здесь мы снимем такое ограничение, используя приемы из $[5,11]$. Пусть вещественное число $\mu$ лежит вне контуров $|\lambda|=r$ и $\gamma_{n}$. Положим $g=(L-\mu E) \varphi$. Тогда $\varphi=R_{\mu} g$, и $g=(L-\lambda E) \varphi+(\lambda-\mu) \varphi$, откуда

$$
R_{\lambda} \varphi=-\frac{\varphi}{\lambda-\mu}+\frac{R_{\lambda} g}{\lambda-\mu} .
$$

Используя (13), аналогично $[5,11]$, для $u(x, t)$ получим следующее представление:

$$
u(x, t)=u_{0}(x, t)+u_{1}(x, t),
$$

где

$$
\begin{aligned}
& u_{0}(x, t)=-\frac{1}{2 \pi i}\left(\int_{|\lambda|=r}+\sum_{n \geqslant n_{0}} \int\right) \frac{R_{\lambda}^{0} g}{\lambda-\mu_{0}} e^{\lambda t} d \lambda, \\
& u_{1}(x, t)=-\frac{1}{2 \pi i}\left(\int_{|\lambda|=r}+\sum_{n \geqslant n_{0}} \int\right) \frac{R_{\lambda} g-R_{\lambda}^{0} g}{\lambda-\mu_{0}} e^{\lambda t} d \lambda,
\end{aligned}
$$

$R_{\lambda}^{0}$ - резольвента оператора $L_{0}$.

Используя соотношение, аналогичное $(13)$, для $R_{\lambda}^{0}$, имеем

$$
\frac{R_{\lambda}^{0} g}{\lambda-\mu}=\frac{R_{\mu}^{0} g}{\lambda-\mu}+R_{\lambda} \widetilde{\varphi},
$$


где $\widetilde{\varphi}=R_{\mu}^{0} g$, откуда

$$
u_{0}(x, t)=-\frac{1}{2 \pi i}\left(\int_{\lambda \mid=r}+\sum_{n \geqslant n_{0}} \int_{\gamma_{n}}\right) R_{\lambda}^{0} \widetilde{\varphi} e^{\lambda t} d \lambda
$$

при этом $\widetilde{\varphi}(x)$ удовлетворяет условиям (5). Таким образом, $u_{0}(x, t)$ есть формальный ряд задачи (1)-(2) с $q_{1}(x)=0$ и $\widetilde{\varphi}(x)=R_{\mu}^{0} g$ вместо $\varphi(x)$ (т.е. эталонной задачи). Согласно теореме 1 этот ряд суммируется и $u_{0}(x, t)$ определяется формулой $(6)$.

Исследуем сходимость и возможность почленного дифференцирования ряда в (15). Имеем

$$
\int_{\gamma_{n}} \frac{R_{\lambda} g-R_{\lambda}^{0} g}{\lambda-\mu} e^{\lambda t} d \lambda=\frac{\left(g, z_{n}\right)}{\left(\lambda_{n}-\mu\right)\left(y_{n}, z_{n}\right)} y_{n}(x) e^{\lambda_{n} t}-\frac{\left(g, z_{n}^{0}\right)}{\left(\lambda_{n}^{0}-\mu\right)\left(y_{n}^{0}, z_{n}^{0}\right)} y_{n}^{0}(x) e^{\lambda_{n}^{0} t} .
$$

Лемма 8. Для любой функиии $g \in L_{2}[0,1] u \psi_{k n}, k=1,2$, из теоремы 2 имеют место соотношения

$$
\left(g(x), \psi_{k n}\right)=\frac{\alpha_{n}}{n} .
$$

Доказательство. Так как $\left(g(x), b(x) e^{ \pm \lambda_{n}^{0} x}\right)=\left(\widetilde{g}(x), e^{ \pm 2 \pi n i x}\right)=\alpha_{n}, \widetilde{g}(x) \in L_{2}[0,1]$, то для $\psi_{1 n}$ утверждение леммы очевидно. Далее,

$$
\begin{aligned}
\left(g(x), b(x) \int_{0}^{x} b(x, t) e^{ \pm 2 \pi n i t} d t\right)=\int_{0}^{1} g(x) \overline{b(x)} d x \int_{0}^{x} \overline{b(x, t)} e^{\mp 2 \pi n i t} d t= & \\
& =\int_{0}^{1} e^{\mp 2 \pi n i t} d t \int_{t}^{1} \widetilde{g}(x) \overline{b(x, t)} d x=\alpha_{n} ;
\end{aligned}
$$

здесь $b(x), b(x, t)$ - некоторые непрерывные функции, $\widetilde{g}(x) \in L_{2}[0,1]$. Отсюда следует утверждение леммы и для $\psi_{2 n}$.

Лемма 9. Имеет место соотношение

$$
\frac{\left(g, z_{n}\right)}{\left(\lambda_{n}-\mu\right)\left(y_{n}, z_{n}\right)} y_{n}(x) e^{\lambda_{n} t}=\frac{\left(g, z_{n}^{0}\right)}{\left(\lambda_{n}^{0}-\mu\right)\left(y_{n}^{0}, z_{n}^{0}\right)} y_{n}^{0}(x) e^{\lambda_{n}^{0} t}+\Omega_{n}(x, t) .
$$

При этом при $x \in[0,1]$ и $t \in[-A, A]$, где $A>0$ произвольно, справедливь оченки

$$
\Omega_{n}(x, t)=\frac{\alpha_{n}}{n^{2}}, \quad \frac{\partial}{\partial x} \Omega_{n}(x, t)=\frac{\alpha_{n}}{n}, \quad \frac{\partial}{\partial t} \Omega_{n}(x, t)=\frac{\alpha_{n}}{n} .
$$

Доказательство. Из теоремы 2 получаем (17), где

$$
\begin{aligned}
\Omega_{n}(x, t)=\left(\lambda_{n}^{0}\right)^{-1} e^{\lambda_{n}^{0} t}\left\{\left(g, z_{n}\right)\left[\psi_{1 n}(x)+\psi_{2 n}(x)+O\left(\frac{1}{n}\right) y_{n}^{0}(x)\right]+\right. & \\
& \left.+\left[\left(g, \psi_{1 n}\right)+\left(g, \psi_{2 n}\right)\right] y_{n}^{0}(x)\right\}+O\left(\frac{1}{n^{3}}\right) .
\end{aligned}
$$

Отсюда, из леммы 8 и соотношения $\left(g, z_{n}\right)=\alpha_{n}$ следуют оценки (18).

Из (15), (16) и леммы 9 получаем следующую лемму.

Лемма 10. Ряд в (15) и ряды, полученные из него почленным дифференцированием по $x$ u $t$, равномерно сходятся по $x \in[0,1] u t \in[-A, A]$, где $A>0$ произвольно.

Теорема 3. Если $q_{k}(x)$ - комплекснозначные функции, $q_{k}(x) \in C^{1}[0,1]$, а функция $\varphi(x) \in$ $C^{1}[0,1]$ удовлетворяет условиям (3), то классическое решение задачи (1)-(2) существует и имеem вид

$$
u(x, t)=u_{0}(x, t)+u_{1}(x, t),
$$


где $u_{0}(x, t)$ задается формулой (6) из теоремы 1 при замене $\varphi$ на $\widetilde{\varphi}=R_{\mu}^{0} g, g=L \varphi-\mu \varphi, u_{1}(x, t)$ определена по формуле (15).

Доказательство. По теореме 1 и лемме $10 u(x, t)$ непрерывно дифференцируема по обеим переменным. Легко проверяется, что $u(x, t)$ удовлетворяет условиям (2). Докажем, что $u(x, t)$ удовлетворяет уравнению (1). Обозначим через $D u$ следующее дифференциальное выражение

$$
D u=\frac{\partial u(x, t)}{\partial t}-\frac{\partial u(x, t)}{\partial x} .
$$

Тогда имеем $D u_{0}=q_{1}(x) u_{0}$. Далее,

$$
\begin{gathered}
\left(R_{\lambda} g(x)\right)^{\prime}=-q_{1}(x) R_{\lambda} g(1-x)-q_{2}(x) R_{\lambda} g(x)+\lambda R_{\lambda} g(x)+g(x), \\
\left(R_{\lambda}^{0} g(x)\right)^{\prime}=-q_{2}(x) R_{\lambda}^{0} g(x)+\lambda R_{\lambda}^{0} g(x)+g(x),
\end{gathered}
$$

откуда

$$
D u_{1}=q_{1}(x) u(1-x, t)+q_{2}(x) u_{1}(x, t) .
$$

Следовательно,

$$
D u=D u_{0}+D u_{1}=q_{1}(x) u(1-x, t)+q_{2}(x)\left(u_{0}(x, t)+u_{1}(x, t)\right)=q_{1}(x) u(1-x, t)+q_{2}(x) u(x, t) .
$$

Теорема доказана.

\section{СПИСОК ЛИТЕРАТУРЫ}

1. Андреев А. А. Об аналогах классических краевых задач для одного дифференциального уравнения второго порядка с отклоняющимся аргументом// Диффер. уравн. - 2004. - 40, № 5. - С. 1126-1128.

2. Баскаков А. Г., Ускова Н. Б. Метод Фурье для дифференциальных уравнений первого порядка с инволюцией и группы операторов// Уфим. мат. ж. - 2018. - 10, № 3. - С. 11-34.

3. Бурлуцкая М. Ш. Асимптотические формулы для собственных значений и собственных функций функционально-дифференциального оператора с инволюцией// Вестн. Воронеж. гос. ун-та. Сер. Физ. Мат. - 2011. - 2. - C. $64-72$.

4. Бурлуцкая М. Ш. О смешанной задаче для уравнения первого порядка с инволюцией и с периодическими краевыми условиями// Ж. вычисл. мат. мат. физ. - 2014. - 54, № 1. - С. 3-12.

5. Бурлуцкал М. Ш. Классическое и обобщенное решение смешанной задачи для системы уравнений первого порядка с непрерывным потенциалом// Ж. вычисл. мат. мат. физ. - 2019. - 59, № 3. C. $380-390$.

6. Бурлуцкая М. Ш., Хромов А. П. Классическое решение для смешанной задачи с инволюцией// Докл. PAH. - 2010. - 435, № 2. - C. 151-154.

7. Бурлуцкая М. Ш., Хромов А. П. Метод Фурье в смешанной задаче для уравнения первого порядка с инволюцией// Ж. вычисл. мат. мат. физ. - 2011. - 51, № 12. - С. 2233-2246.

8. Бурлуцкал М. Ш., Хромов А. П. Теорема Штейнгауза о равносходимости для функциональнодифференциальных операторов// Мат. заметки. - 2011. - 90, № 1. - С. 22-33.

9. Бурлуцкая М. Ш., Хромов А. П. Смешанная задача для простейшего гиперболического уравнения первого порядка с инволюцией// Изв. Саратов. ун-та. Нов. сер. Сер. Мат. Мех. Информ. - 2014. 14, № 1. - C. 10-20.

10. Бурлуцкал М. Ш., Хромов А. П. Функционально-дифференциальные операторы с инволюцией и операторы Дирака с периодическими краевыми условиями// Докл. РАН. - 2014. - 454, № 1. - С. 15-17.

11. Бурлуикая М. Ш., Хромов А. П. Резольвентный подход для волнового уравнения// Ж. вычисл. мат. мат. физ. - 2015. - 55, № 2. - С. 51-63.

12. Бурлуцкая М. Ш., Курдюмов В. П., Луконина А. С., Хромов А. П. Функциональнодифференциальный оператор с инволюцией// Докл. РАН. - 2007. - 414, № 4. - С. 1309-1312.

13. Вагабов А. И. Введение в спектральную теорию дифференциальных операторов. - Ростов-на-Дону: Изд-во РГУ, 1994.

14. Хромов А. П. Об обращении интегральных операторов с ядрами, разрывными на диагоналях// Мат. заметки. - 1998. - 64, № 6. - С. 932-949.

15. Baskakov A. G., Krishtal I. A., Romanova E. Yu. Spectral analysis of a differential operator with an involution// J. Evolut. Equations. - 2017. — 17. - P. 669-684. 
16. Kritskov L. V., Sarsenbi A. M. Spectral properties of a nonlocal problem for a second-order differential equation with an involution// Differ. Equations. — 2015. - 51, № 8. - P. 984-990.

Белова Диана Владиславовна

Воронежский государственный университет

E-mail: dianabelova123@yandex.ru 